\title{
A CASE REPORT OF PREGNANCY WITH EISENMENGER SYNDROME， A RARE CASE
}

\section{Dr Hafsa Vohra}

Dr Jalpa Rathod*
Assistant Professor, Civil Hospital Ahmedabad, Dept Of Obgy. Third Year Resident, Civil Hospital, Ahmedabad, Dept Of Obgy. ${ }^{*}$ Corresponding Author

ABSTRACT Pregnancy complicated with Eisenmenger syndrome is associated with high risk to the mother and the fetus. There is approximately $50 \%$ risk of sudden maternal death, frequently occurring a few days postpartum and the overall fetal wastage is reported to be up to $75 \%$. Patients with Eisenmenger syndrome are advised to refrain from pregnancy or to terminate pregnancy by the end of first trimester itself. Management of these patients requires a coordinated multi-specialist care when such pregnancies reach a stage where safe termination is not advisable. However, inspite of all the risks, a few patients delivered successfully with a good maternal and neonatal outcome. Here we report a case of a 20 years old primi patient with 36 weeks gestational age and known case of Ventricular Septal Defect with Eisenmenger syndrome and Tricuspid Regurgitation and Pulmonary Hypertension. Her doppler study suggestive of utero placental insuffiency with reversal of umbilical artery flow. Patient was delivered normally with full support of oxygen with good neonatal outcome. Patient and her baby were transferred to cardiology department and patient was discharged on post partum day 5 from cardiology hospital.

\section{KEYWORDS : Eisenmenger Syndrome, Pulmonary Hypertension, Right To Left Shunt.}

\section{INTRODUCTION}

Pregnancy complicated with Eisenmenger syndrome is associated with high risk to the fetus as well as the mother. There is approximately $50 \%$ risk of sudden maternal death, frequently occurring a few days postpartum and the overall fetal wastage is reported to be up to $75 \%$. Patients with Eisenmenger syndrome are advised to refrain from pregnancy or to terminate pregnancy by the end of first trimester itself. Management of these patients requires a coordinated multi-specialist care when such pregnancies reach a stage where safe termination is not advisable.

However, in spite of all the risks, a few patients deliver successfully with a good maternal and neonatal outcome.

\section{CASE REPORT}

We present a case report of a 20 year old Primi patient who came to emergency department at 36 week gestational age with chief complain of lower abdominal pain and breathlessness for 2 days.

Our patient was known case of ventricular septal defect with Eisenmenger syndrome with Severe Tricuspid Regurgitation and Pulmonary Hypertension which was diagnosed at 6 month of amenorrhea Patient was on regular medication (tablet torsemide 1 tablet once a day).

Patient had not taken any ANC care till 6 months when her cardiac condition was detected .At 9 months of amenorrhea, USG Antenatal doppler study was done which was s/o utero placental insuffiency with reversal of umbilical artery blood flow. Fetal cardiac condition were s/o moderate cardiomegaly, left axis deviation, veno arterial and ventriculo-atrial concordance noted, right atrium and right ventricle enlarged ,right ventricular hypertrophy, pulmonary artery mildly dilated with pulmonary edema. Patient was then referred to Civil Hospital Ahmedabad

On examination patient was of normal stature and poorly built, weight of $47 \mathrm{~kg}$. Examination revealed edema, clubbing on all four limbs and vitals were stable with pulse rate 92 beats/min and blood pressure $130 / 90 \mathrm{mmhg}$ in right upper limb. Her respiratory rate was $35 /$ minute and JVP was raised. On CVS examination she had thrills over apex pansystolic murmur with S2 loud. Apex beat was shifted to mid clavicular line and left paracentral heave was present, Respiratory examination revealed bilateral vesicular breath sounds in all lungs fields. Abdominal examination uterus 30 32 weeks size relaxed with cephalic presentation with fetal heart rate 140 beats/minute. On per vaginal examination cervical os was 2 finger loose, $30 \%$ effaced, membrane was present with station -2 .

On investigation $\mathrm{Hb} 18.5 \mathrm{gm} \%$, hematocrit 61.50\% ECG showed irregular rhythm with ventricular hypertrophy . Cardiology consultation was obtained after she was put on tablet sildenafil, dytor and spironolactone. Cardiologist opinion was taken and advice was to shorten the second stage of labour .Patient was put on complete bed rest with 45 degree inclination with oxygen with venti mask @5 L/ min. Injection Betamethasone for fetal lung maturity was given. Bacterial endocarditis prophylaxis in the form of injectable antibiotics was started.

Labour was augmented by Intravenous oxytocin and patient delivered normally. A pre term female child of $1 \mathrm{~kg}$ delivered liqour was clear and adequate and baby cried soon after birth. Maturity was 32 weeks and Apgar score was 5/6/7.

Intrapartum period was uneventful. and input and output of IV fluids was monitored strictly. Injectable antibiotics were given. On $1^{\text {st }}$ post-partum day, patient was vitally stable. Patient was shifted in ICU for post partum monitoring. Respiratory examination revealed bilateral crepitations. . In ICU she was administered $\mathrm{O} 2$ inhalation via venti mask, injectable antibiotics. Uteruswas well contracted with no post partum hemorrhage. Her condition was stable and then she was transferred to cardiology hospital on post partum day 2 for further management where she was managed conservatively and started on tablet torsemide, tablet spironolactone and tablet sildenafil. Patient was discharged on post partum day 5 from cardiology hospital. Patient was advised regarding contraceptive methods for future.

\section{DISCUSSION}

Patients with Eisenmenger syndrome have Pulmonary Hypertension with right to left or bidirectional shunt through either an open ASD or VSD. In these patients increase in pulmonary pressure or decrease in pulmonary vascular resistance may cause right to left shunting and arterial blood oxygen desaturation.

Outcome of pregnancy in patients with Eisenmenger syndrome is very poor. Due to chronic slow progressive 
hypoxemia with central cyanosis, adult patients with Eisenmenger syndrome suffer from a complex and multisystemic disorder including coagulation disorders, renal dysfunction, heart failure, reduced quality of life and premature death. Mortality is approximately $20-50 \%$ and total fetal wastage is also high. Despite the best cardiac and obstetrical care in these patients there is a high chance of maternal mortality in the post partum period due to irreversible cardiovascular collapse. In addition to the general measures of the pregnant cardiac patients described above, anti coagulation with heparin to avoid the formation of micro thrombi in the pulmonary circulation and new selective pulmonary vasodilators should be used for Eisenmenger syndrome. Epidural narcotics can be used for pain relief during labor. To maintain SVR above that of the pulmonary artery and to avoid right to left shunting it may be necessary to use IV fluids and peripheral vasodilators during delivery and during puerperium.

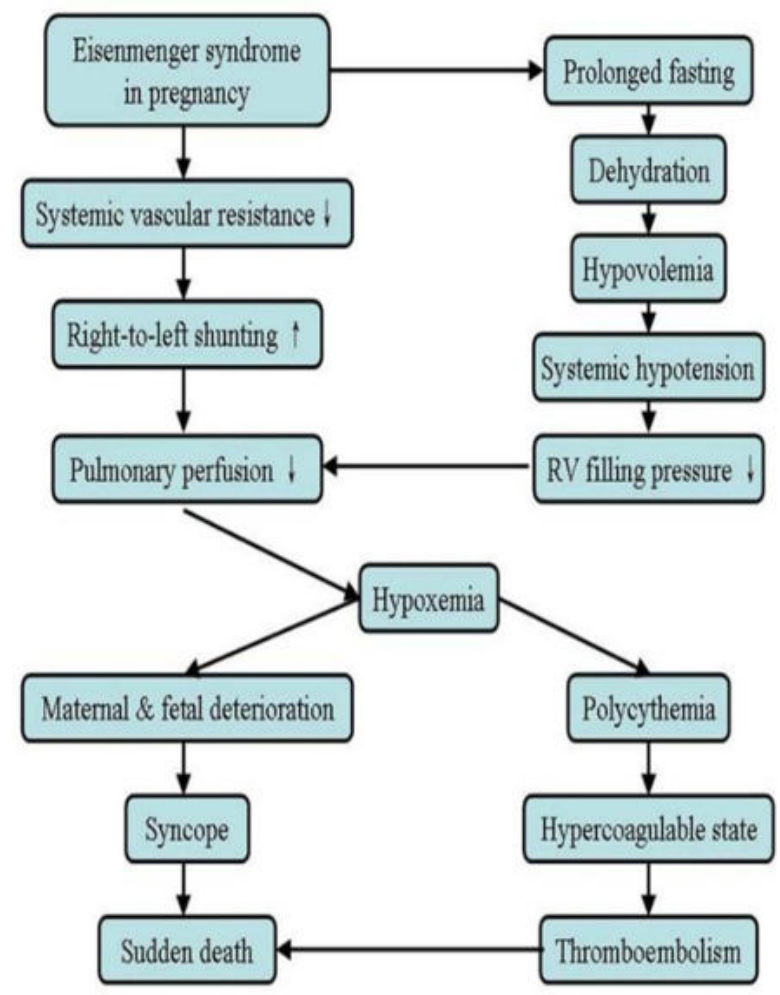

Figure 1 Schematic representation of pathophysiology of Eisenmenger syndrome.

\section{CONCLUSION}

In conclusion all though pregnancy should be discouraged women with Eisenmenger syndrome. In cases of pregnancy in such case it can be successful with coordination of multispecialty doctors like Cardiologist, Obstetricians and Neonatologist and proper monitoring help in avoiding any adverse maternal or fetal outcome. This case may help in giving a hope to all such pregnancy complicated with Eisenmenger syndrome with advanced gestational age where termination is not possible.

\section{REFERENCES}

1. Williams Obstetrics; 24th edition;2014; Mc Graw Hill Medical;1125-1146

2. Arias' practical guide to high risk pregnancy and delivery, $5^{\text {th }}$ edition

3. Miller LD Eisenmenger syndrome and pregnant patient JOGN nursing volume 12 , issue 31983 , pages 175-180 ISSN 0090-0311 